\title{
Spin-exchange of axially symmetric Mu states in polycrystalline media
}

\author{
J. Piroto Duarte ${ }^{\mathrm{a}, \mathrm{b}, *}$, R.C. Vilão ${ }^{\mathrm{b}}$, H.V. Alberto $^{\mathrm{b}}$, J.M. Gil $^{\mathrm{b}}$, N. Ayres de Campos ${ }^{\mathrm{b}}$ \\ ${ }^{a}$ E.S.T.S.C., Polytechnic Institute of Coimbra, P-3040-854 Coimbra, Portugal \\ b CEMDRX, Department of Physics, University of Coimbra, P-3004-516 Coimbra, Portugal
}

\section{A R T I C L E I N F O}

Keywords:

Muon spin rotation

Muonium

Spin exchange

\begin{abstract}
A B S T R A C T
A theoretical discussion of the features expected for the longitudinal-field relaxation signal of axially symmetric $\mathrm{Mu}$ states in polycrystalline samples is presented here. It is shown that the muon polarization may be described by the sum of an exponentially relaxed component and a non-relaxed one. Using this approximation, an analysis framework that allows extracting the spin-flip rate from polycrystalline data is also sketched.
\end{abstract}

(c) 2008 Elsevier B.V. All rights reserved.

\section{Introduction}

The scattering of a paramagnetic species and the bound electron in a Mu state strongly influences the time evolution of the muon spin, constituting what is termed in $\mu$ SR literature as spin-exchange (SE) dynamics. The effect of the scattering is conveyed to the muon via the hyperfine interaction, imparting a depolarization to the $\mu$ SR signal which directly relates with the time structure of the physical phenomena producing the scattering. In this way, $\mu S R$ is used to study numerous dynamical effects, from charge transport in semiconductors to muon stopping ranges in gases.

The $\mu$ SR signal expected for a Mu state undergoing SE has been the subject of several theoretical studies dating from the birth of $\mu S R$ to our days (see e.g. Refs. [1-3]), in which the transverse- and longitudinal-field (LF) signals in the slow and fast spin-flip regimes became well established using different theoretical approaches. Those results consider always that the $\mathrm{Mu}$ state either has an isotropic hyperfine interaction, or that when the hyperfine tensor is anisotropic, the three principal axes of the tensor have known orientation relative to the applied magnetic field and the direction along which the muon polarization is being observed.

Recently, the investigation of local charge conduction in molecular organic semiconductors [4-6] forced the consideration of SE dynamics for axially symmetric Mu states in polycrystalline media, for which the known SE muon polarization functions cannot be directly applied. The need was prompted by the fact that these novel materials cannot be grown in single crystals large enough to use in a $\mu$ SR experiment, nor are soluble enough to use

\footnotetext{
* Corresponding author at: E.S.T.S.C., Polytechnic Institute of Coimbra, P-3040854 Coimbra, Portugal. Tel.: +351 239 802430; fax: +351 239813395.

E-mail address: piroto@ci.uc.pt (J.P. Duarte).
}

liquid samples where the anisotropy of the hyperfine interaction is averaged out.

We present here a theoretical discussion of the features expected for the LF signal of axially symmetric Mu states in polycrystalline samples, and setup an analysis framework that allows extracting the spin-flip rate from that type of data.

\section{Spin exchange in polycrystalline media}

In LF geometry, the non-oscillating time-dependent muon polarization of a single anisotropic $\mathrm{Mu}$ state undergoing random spin-flip events at a rate $\lambda_{\mathrm{SF}}$ is an exponentially damped function [7]

$P(t)=a_{0} \mathrm{e}^{-\lambda_{L} t}$

with a relaxation rate given by [8]

$\lambda_{L}=\lambda_{\mathrm{SF}} \sum_{n, m=1}^{4} \frac{\omega_{n m}^{2}}{\lambda_{\mathrm{SF}}^{2}+\omega_{n m}^{2}} a_{n m}$

where $\omega_{n m}$ are the Mu hyperfine transition frequencies, and $a_{n m}$ are the expected amplitudes of the corresponding precessions along the observation direction (see e.g. Ref. [9]). $a_{0}$ is the nonoscillating amplitude

$a_{0}=\sum_{n=1}^{4} a_{n n}$

in the slow spin-flip regime $\left(\lambda_{\mathrm{SF}} \ll \omega_{n m}\right)$ and

$a_{0}=1$

in the fast spin-flip regime $\left(\lambda_{\mathrm{SF}} \gg \omega_{n m}\right)$ [8]. For an axially symmetric state, the values of $\lambda_{L}$ and $a_{0}$, besides depending of the hyperfine parameters of the state and the intensity of the applied field, are both a function of the angle $\theta$ defined between 


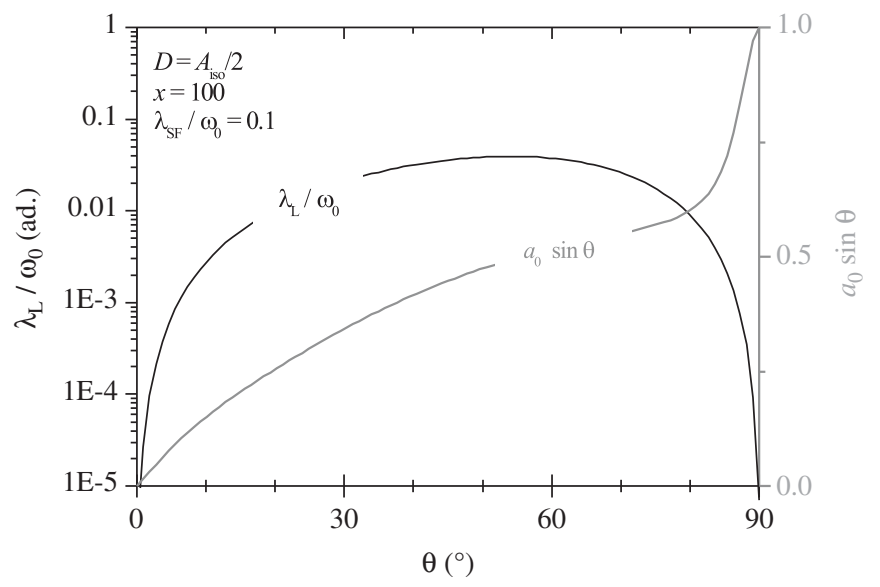

Fig. 1. Orientation dependence of the LF relaxation rate and the (solid angle weighted) non-oscillating amplitude for an axially symmetric Mu state with a large dipolar parameter undergoing slow spin flip, following calculations performed with Eqs. (2) and (3) using numerical solutions for the orientation dependent hyperfine transition frequencies and corresponding amplitudes. Here, $A_{\text {iso }}$ and $D$ are the hyperfine parameters of the state, $\omega_{0}=2 \pi A_{\text {iso }}$, and $x=B / B_{0}$ is the reduced field, where $B_{0}=\omega_{0} /\left(\gamma_{\mathrm{e}}+\gamma_{\mu}\right)$ and $\gamma_{\mathrm{e}}$ and $\gamma_{\mu}$ are the gyromagnetic ratios of the electron and the muon.

the hyperfine symmetry axis and the magnetic field. The variation is strong (Fig. 1), especially if the state has large dipolar character; for the same spin-flip rate, $\lambda_{L}$ typically covers several orders of magnitude over the $\left[0^{\circ}, 90^{\circ}\right]$ interval.

In a fully non-oriented polycrystalline sample, where a random distribution of $\theta$ values sampling all possible directions exists, this results in an observed muon polarization which is the sum of exponential functions over a broad range of relaxation rates, weighted by the solid angle factor $\sin \theta$ and the non-oscillating amplitude $a_{0}$ :

$P(t)=\int_{0}^{\pi / 2} a_{0}(\theta) \mathrm{e}^{-\lambda_{L}(\theta) t} \sin \theta \mathrm{d} \theta$

This is a time dependence which, due to the specific angular variations of $\lambda_{L}$ and the product $a_{0} \times \sin \theta$ (see Fig. 1), will have two main contributions. The first, corresponding to the middle range of $\theta$ angles, is an approximately exponential function with relaxation rate with the same order of magnitude as the spin-flip rate. The second, originated by the upper range of $\theta$ angles, has a very small relaxing character, which in a frequency-limited $\mu S R$ experiment will not be distinguished from a purely non-relaxing signal. It is therefore possible to assume that

$\int_{0}^{\pi / 2} a_{0}(\theta) \mathrm{e}^{-\lambda_{L}(\theta) t} \sin \theta \mathrm{d} \theta \approx \tilde{a} \mathrm{e}^{-\tilde{\lambda} t}+\tilde{b}$

i.e. that the LF muon polarization of an axially symmetric muonium state undergoing SE dynamics in a polycrystalline sample is given by the approximated polarization function

$\tilde{P}(t)=\tilde{a} \mathrm{e}^{-\tilde{\lambda} t}+\tilde{b}$,

where $\tilde{a}, \tilde{\lambda}$, and $\tilde{b}$ are functions of the hyperfine parameters $A_{\text {iso }}$ and $D$ of the state, of the spin-flip rate $\lambda_{\mathrm{SF}}$, and of the external field $B$.

\section{Analysis framework}

Using the approximation of Eq. (7), the analysis of LF data regarding axially symmetric muon states in polycrystalline media becomes a matter of first analyzing the time-dependent data with a phenomenological fit function

$P_{f i t}(t)=a \mathrm{e}^{-\lambda t}+b$

and, given the hyperfine parameters $A_{\text {iso }}$ and $D$ of the state, and the applied field $B$, obtain the spin-flip rate $\lambda_{\mathrm{SF}}$ that provides the best simultaneous match between the measured fit parameters $(a, \lambda, b)$, and the expected values $(\tilde{a}, \tilde{\lambda}, \tilde{b})$ calculated from $A_{\text {iso }}, D$, $\lambda_{\mathrm{SF}}$, and $B$.

The way how the quantities $(\tilde{a}, \tilde{\lambda}, \tilde{b})$ are calculated from $A_{\text {iso }}, D$, $\lambda_{\mathrm{SF}}$ and $B$ must be based in a method that seeks the triplet $(\tilde{a}, \tilde{\lambda}, \tilde{b})$ which produces an approximation $\tilde{P}(t)$ that is the nearest possible to the full polarization function $P(t)$, Eq. (5), obtained from the values of $A_{\mathrm{iso}}, D, \lambda_{\mathrm{SF}}$, and $B$ given. This may be done using a figure of merit $q$ defined in the sense of minimum squared deviations between $\tilde{P}(t)$ and $P(t)$ over the experimental time window $\left[0, t_{\max }\right]$ of a $\mu \mathrm{SR}$ histogram. This figure, chosen to read explicitly

$q(\tilde{a}, \tilde{\lambda}, \tilde{b})=\frac{1}{t_{\max }} \int_{0}^{t_{\max }}[P(t)-\tilde{P}(t)]^{2} \mathrm{~d} t$

was found to be easily minimized using a fast numerical minimization algorithm and a version of Eq. (9) algebraically manipulated to improve computation time [10] (refer also to the appendix). The translation procedure of $A_{\mathrm{iso}}, D, \lambda_{\mathrm{SF}}$, and $B$ in the secondary parameters $(\tilde{a}, \tilde{\lambda}, \tilde{b})$ is therefore similar to parameter estimation by chi-square fitting. Fig. 2 illustrates the results of this procedure for an axially symmetric state in a range of $\lambda_{\mathrm{SF}}$ rates spanning from near zero up to values comparable to the hyperfine frequencies.

As it may be perceived from Fig. 2, the approximation becomes worse as the ratio $\lambda_{\mathrm{SF}} / A_{\text {iso }}$ gets larger, suggesting that near the transition from slow to fast spin flip it may cease to be valid. In order to clarify this point, extensive simulations were conducted in a variety of situations of interest to $\mu \mathrm{SR}$, namely with $A_{\text {iso }} \in[0,500] \mathrm{MHz}, D \in\left[0, A_{\mathrm{iso}}\right], \lambda_{\mathrm{SF}} \in\left[0, A_{\text {iso }}\right]$ and $B$ assuming lowto Paschen-Back field limits. In those simulations, it was found that the value of $q$, computed in a regular $\mu$ SR time window, remained always well below the time-integrated experimental error due to Poisson statistics for a typical 5 million events experiment. It becomes therefore plausible to assume that, in typical experimental conditions, approximation (6) always holds.

The simulations also analyzed the shape of the $q$-surface around its minimum to ascertain the uniqueness of the $(\tilde{a}, \tilde{\lambda}, \tilde{b})$ triplet. The surface was seen to be always well defined, becoming

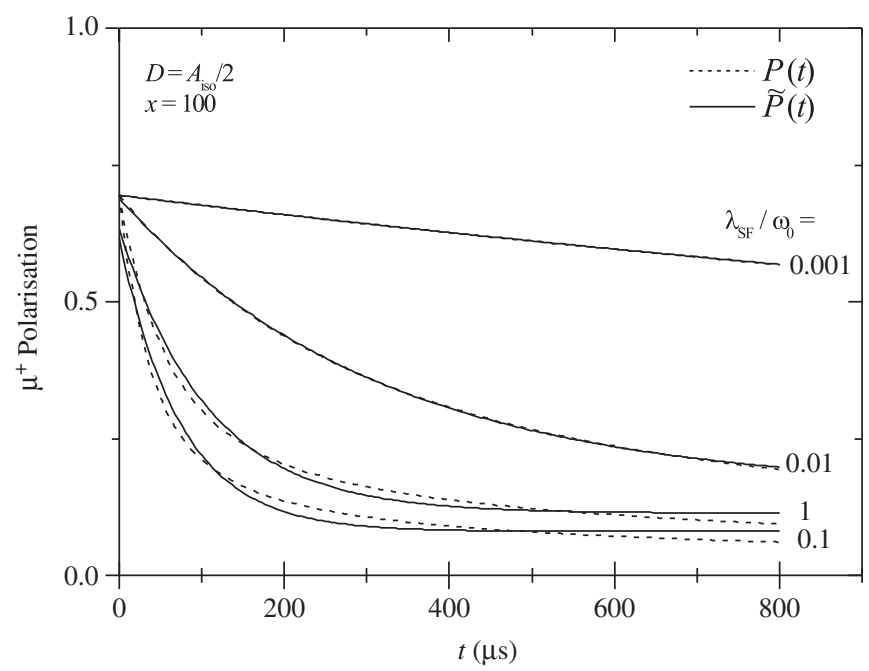

Fig. 2. Comparison between $P(t)$, the exact function for the muon polarization, and the approximation $\tilde{P}(t)$ obtained with the method sketched here, using a set of different spin-flip rates. 
more pronounced as the ratios $\lambda_{\mathrm{SF}} / A_{\mathrm{iso}}, D / A_{\mathrm{iso}}$, and the absolute value of $B$ increased. This sharpening correlates with the worsening of the approximation, a behavior which was to be expected since small changes in the values of $A_{\mathrm{iso}}, D, \lambda_{\mathrm{SF}}$, and $B$ when the deviation between $\tilde{P}(t)$ and $P(t)$ is already considerable should of course produce a larger effect.

\section{Conclusions}

The analysis of the expected $\mu$ SR signal in LF geometry for an axially symmetric muonium state in polycrystalline media, taking into consideration the particular features of the orientation dependence of the relaxation rate and non-oscillating amplitude, show that the time-dependent muon polarization may be described as a simple sum of two components, one relaxed and other non-relaxed. This result can be used to extract the spin-flip rate from the measurements using the method presented here, which involves a phenomenological fit of the time-dependent data with the two component description, and the translation of the fitted parameters to a spin-flip rate by the minimization of a properly chosen figure of merit.

\section{Acknowledgments}

The assistance of all LMU staff at PSI is gratefully acknowledged. This work was performed at the Swiss Muon Source, Paul Scherrer Institut, Villigen, Switzerland, and partially supported by the European Commission under the 6th Framework Programme through the Key Action: Strengthening the European Research Area, Research Infrastructures, Contract no. RII3-CT-2004-505925. We also thank the support of the Portuguese Foundation for Science and Technology (FCT) through Grant no. POCTI-SFA-2-30 and the PRODEP III program under action 5.3.

\section{Appendix}

The version of Eq. (9) used in the minimization routine referred in Section 3 is

$q(\tilde{a}, \tilde{\lambda}, \tilde{b})=\frac{1}{t_{\max }}\left(q_{1}+q_{2}+q_{3}\right)$,

where the quantities $q_{1}, q_{2}$, and $q_{3}$ read explicitly

$$
\begin{aligned}
q_{1}= & \int_{0}^{\pi / 2} a_{0}(\theta) \sin \theta \int_{0}^{\pi / 2} a_{0}\left(\theta^{\prime}\right) \sin \theta^{\prime} \\
& \times \frac{1-\mathrm{e}^{-\left(\lambda_{L}(\theta)+\lambda_{L}\left(\theta^{\prime}\right) t_{\max }\right.}}{\lambda_{L}(\theta)+\lambda_{L}\left(\theta^{\prime}\right)} \mathrm{d} \theta^{\prime} \mathrm{d} \theta, \\
q_{2}= & -2 \int_{0}^{\pi / 2} a_{0}(\theta) \sin \theta \\
& \times\left\{\tilde{a} \frac{1-\mathrm{e}^{-(\lambda(\theta)+\tilde{\lambda}) t_{\max }}}{\lambda(\theta)+\tilde{\lambda}}+\tilde{b} \frac{1-\mathrm{e}^{-\lambda(\theta) t_{\max }}}{\lambda(\theta)}\right\} \mathrm{d} \theta, \\
q_{3}= & \tilde{a}^{2} \frac{1-\mathrm{e}^{-2 \tilde{\lambda} t_{\max }}}{2 \tilde{\lambda}}+\tilde{b}^{2} t_{\max }+2 \tilde{a} \tilde{b} \frac{1-\mathrm{e}^{-\tilde{\lambda} t_{\max }}}{\tilde{\lambda}} .
\end{aligned}
$$

The angular integrations in these expressions were performed in our program using a Simpson-rule algorithm.

\section{References}

[1] R.E. Turner, et al., Phys. Rev. A 41 (1990) 1505.

[2] M. Celio, P.F. Meier, Phys. Rev. B 27 (1983) 1908.

[3] M. Senba, J. Phys. B: At. Mol. Opt. Phys. 23 (1990) 4051.

[4] J. Piroto Duarte, et al., Phys. Rev. B 73 (2006) 075209.

[5] J. Piroto Duarte, et al., Physica B 326 (2003) 94.

[6] J. Piroto Duarte, et al., Physica B 374-375 (2006) 426.

[7] M. Senba, Phys. Rev. A 62 (2000) 042505.

[8] M. Senba, J. Phys. B: At. Mol. Opt. Phys. 34 (2001) 4437.

[9] B.D. Patterson, Rev. Modern Phys. 60 (1988) 69.

[10] J. Piroto Duarte, Ph.D. Thesis, 2007 (unpublished). 